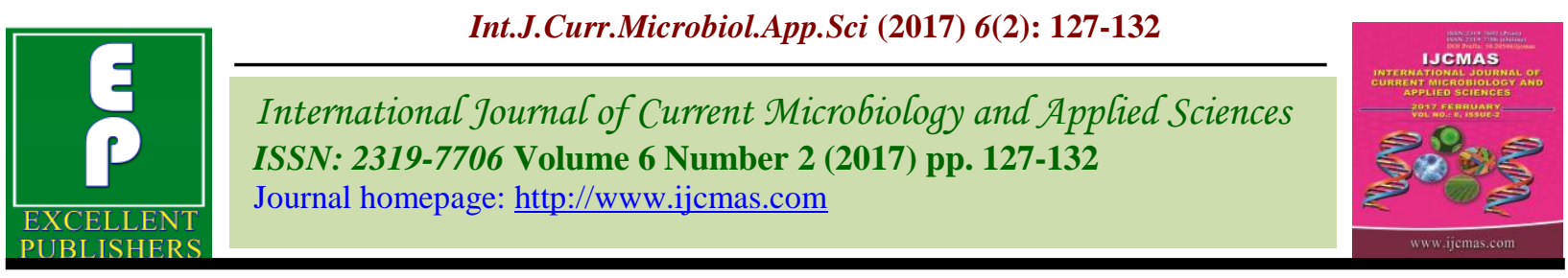

Original Research Article

http://dx.doi.org/10.20546/ijcmas.2017.602.018

\title{
Mycological Profile of Chronic Suppurative Otitis Media in a Tertiary Care Hospital in South India
}

\author{
Sahira Haneefa*, Sathya Bhama and Jyothi Rajahamsan \\ Department of Microbiology, Government Medical College, Thiruvananthapuram, India \\ *Corresponding author
}

A B S T R A C T

\section{Keywords}

Chronic suppurative otitis media, Otomycosis, Aspergillus, Candida.

Article Info

Accepted:

05 January 2017

Available Online:

10 February 2017
Chronic suppurative otitis media (CSOM) is chronic inflammation of middle ear and mastoid cavity with recurrent ear discharge lasting for more than 6months. Studies are mainly done on the bacterial aetiology of CSOM, but very little is known about the fungal aspects of such cases. Otomycosis is increasing in the recent years because of the indiscriminate use of broad-spectrum antibiotics, corticosteroids, and an increase in the number of immune deficiency disorders. The study aimed to find out the fungal agents responsible for causing CSOM among the patients who attended the Department of ENT in our hospital. 170 patients having chronic suppurative otitis media of all age group and both sexes were selected for study from April 2014 to December 2015. Ear discharge was collected under aseptic precautions using sterile cotton swabs and fungal culture was done. A total of 170 patients who were clinically diagnosed with CSOM were included in this study. Of which 104(61\%) were females and 66(39\%) were males. Fungus isolated from $87(51.2 \%)$ cases. Main fungal isolates were Aspergillus species 67(77\%) followed by Candida species $10(11.4 \%)$. CSOM with otomycosis produce serious complication and treatment failure. So fungal infection should be suspected if the discharging ear is not responding with antibiotics and fungal culture should be done in refractory cases before starting antibiotic therapy.

\section{Introduction}

Otitis Media is a persistent, insidious and dangerous disease because of multiple aetiology and fatal complications. The importance of Otitis Media lies in its refractoriness to treatment and its complications (Rama Rao et al., 1980). It is one of the major cause of conductive deafness and delayed development of speech in children. Chronic suppurative otitis media (CSOM)is the inflamation of middle ear and mastoid cavity with recurrent ear discharge through perforated tympanic membrane (Michael Gleeson et al., 2008).
CSOM is mainly caused by bacteria, so studies are mainly focused on finding the bacterial aetiology. Most commonly isolated aerobic bacteria in CSOM are Pseudomonas aeruginosa, Staphylococcus aureus, Klebsiella, Streptococcus pyogenes etc. The irrational use of broad spectrum antibiotics, use of steroids and immunodeficiency disorders favours the secondary infection by fungi. Presence of moisture in ear canal also favours fungal infection by Candida, Aspergillus, etc. 
The microbiology investigations help in reducing the active infection of CSOM and thus prevent further serious complications such as mastoiditis and brain abscess.

\section{Materials and Methods}

The main aim and Objective of the study is to identify the fungal aetiological agents of CSOM in patients attended in the Department of ENT, Govt. Medical College, Thiruvananthapuram. A cross sectional study was carried out in Department of Microbiology, Govt. Medical College, Thiruvananthapuram from April 2014December 2015. The study included patients of all ages and both sexes with ear discharge of more than six months duration. Otomycosis which does not have a CSOM association and patients on topical antifungal therapy were excluded.

Data collection made using a proforma for each patient regarding name, age, sex, address. The clinical information like earache, ear discharge, duration of symptoms, predisposing factors, history of recurrence, the treatment taken etc are also included in the proforma. Other medical history like diabetes mellitus, hypertension and tuberculosis were also noted.

Ear discharge was collected under aseptic precautions. Excess discharge was mopped and the external auditory canal cleaned using sterile normal saline. The specimen was then collected using two sterile cotton swabs. One swab was subjected to microscopic examination by $\mathrm{KOH}$ wet mount preparation and the other swab was used for inoculation in Sabouraud's dextrose agar (two tubes). One tube kept at $37^{\circ} \mathrm{c}$ and other at room temperature. Fungal growth obtained on SDA were examined for characteristics like rate of growth, colony morphology, colour of obverse and reverse, diffusible pigment
production(Rippon JW 1988). Fungal growth was examined by a lactophenol cotton blue tease mount preparation. A slide culture was also done in doubtful cases. Lactophenol cotton blue stain is the preferred stain for microscopic examination of fungi in both tease mount and slide culture. Germ tube test and chlamydospore formation were used to differentiate Candida albicans from other Candida species. Antibiotic susceptibility testing by disc diffusion method for Candida was also done (M-44A, CLSI). Antifungal discs used were Fluconazole $(10 \mu \mathrm{g})$, Amphotericin B (100units). Zone size interpretation for Candida species was done as per CLSI guideline.

\section{Results and Discussion}

One hundred and seventy clinically diagnosed cases of active chronic suppurative otitis media attended ENT outpatient department were studied in the Department of Microbiology, Medical College Hospital, Trivandrum. Relevant findings made from the study are as follows.

Table- 1 shows that incidence of otitis media is lower in males compared to females. Out of 170 otitis media patients $66(39 \%)$ were males and $104(61 \%)$ were females. The table also shows that $87(51.2 \%)$ cases were culture positive.

Incidence of otitis media is more common during winter and early spring as shown in table-2.

The table 3 shows that unilateral infection $162(95 \%)$ is more compared to bilateral infection $8(4.7 \%)$ and right ear is more involved than left.

Analysis of symptoms in table 4 shows that otorrhoea was present in $100 \%$ of patients. Itching and earache were the next major 
complaints. It is followed by fever, hearing defect, headache and tinnitus.

Table-5 shows that among the fungal pathogens Aspergillus form the major pathogen $67(77 \%)$ followed by Candida 10(11.4\%) Penicillium 4(4.7\%) Mucor 3 (3.4\%) and Aureobasidium 2(2.3\%).

The highest isolation rate for Aspergillus species was observed in 31-40 age group followed by 21-30 age group as shown in table-6. Majority of the patients were females and $60 \%$ had history of diabetes mellitus.

All Candida species were sensitive to

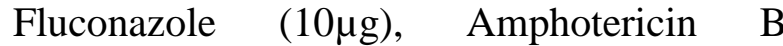
(100units).

The present study is to know the fungal aetological agents of Chronic Suppurative Otitis Media. The results are compared and correlated with the studies conducted by other researchers.

Out of 170 otitis media patients $66(39 \%)$ were males and 104(61\%) were females. The study conducted by Pankti Panchal et al., (2013) and Murat Ozean et al., (2003) shows that females were commonly involved compared to males. Studies done by Pradhan et al., (2003) and B Vishwanatha et al., (2012) reported that otomycosis is more common in males.

In the present study it has been observed that CSOM is more prevalent in persons with age group between 31 and 40 years followed by 21-30. The findings correlated with the studies conducted by Erkan Mustaya et al., (1986) Gulati et al., (1997) and Taneja et al., (1998) Khurana et al., (1998) and Mohanty et al., (1999).

Otitis media cases were more prevalent during winter season and early spring and the present study also confirmed the same and 89(52.4\%) and $56(32.9 \%)$ cases were reported in winter and early spring respectively. The present findings correlated with Charles D. Bluestone (2004). The increased incidence in winter season is mainly attributed to repeated viral / bacterial upper respiratory tract infection

In the present study unilateral infection $162(95 \%)$ is more compared to bilateral infection $8(4.7 \%)$ and right ear is more involved than left. These findings are correlated with the studies conducted by Gulati et al., (1997) and Urmil Mohan et al., (1998). In these cases the percentage of unilateral infection are $80 \%, 86.3 \%$ respectively. Since most of the persons are right handed, chance of introduction of infection to right ear is more common.

Out of 87 culture positive cases, Aspergillus forms the major pathogen $67(77 \%)$ followed by Candida 10(11.4\%) Penicillium 4(4.7\%) and Mucor spp 3(3.4\%) Aureobasidium $2(2.3 \%)$. The fungal aetiology of CSOM was correlated by the studies conducted with the following workers. Urmil Mohan and Neerja Jindal in 1998 done a study on fungal and bacterial flora of chronic suppurative Otitis Media in Punjab. They observed that Aspergillus spp. and Candida albicans are the most common fungal isolates causing CSOM. Another study conducted in Microbiology department, Thiruvananthapuram in 2003 showed that among the fungal pathogens causing CSOM, Aspergillus spp. accounts major contribution followed by Candida albicans. A study conducted by Dr. Sreekumar in ENT department, in Medical College, Thiruvananthapuram (2007) observed that Aspergillus niger and fumigatus are the most common cause of otomycosis. In the study conducted by Loy (2002) on patients with CSOM Aspergillus niger, Aspergillus spp. and Candida spp. were main isolates 16. Studies by Asok et al., and Arya et al., (1966) shows equal distribution of Aspergillus niger and Candida albicans. 
Table.1 Gender wise distribution

\begin{tabular}{|l|c|c|}
\hline Gender & Total number of cases & Number of Culture positives \\
\hline Female & $104(61 \%)$ & $53(61 \%)$ \\
\hline Male & $66(39 \%)$ & $34(39 \%)$ \\
\hline Total & $\mathbf{1 7 0}$ & $\mathbf{8 7}$ \\
\hline
\end{tabular}

Table.2 Season wise distribution

\begin{tabular}{|l|l|}
\hline Month & Number of patients \\
\hline March- June (Summer) & $25(14.7 \%)$ \\
\hline July-October (Spring) & $56(32.9 \%)$ \\
\hline November-February (Winter) & $89(52.4 \%)$ \\
\hline
\end{tabular}

Table.3 Distribution of cases according to ear affected

\begin{tabular}{|l|l|}
\hline Ear affected & Number of patients \\
\hline Right & $90(53 \%)$ \\
\hline Left & $72(42.3 \%)$ \\
\hline Both ears & $8(4.7 \%)$ \\
\hline Total & $\mathbf{1 7 0}$ \\
\hline
\end{tabular}

Table.4 Analysis of symptoms

\begin{tabular}{|l|c|}
\hline Chief complaints & Number of patients and \% \\
\hline Otorrhoea & $170(100 \%)$ \\
\hline Itching & $160(94.1 \%)$ \\
\hline Earache & $156(91.7 \%)$ \\
\hline hearing defect & $32(18.8 \%)$ \\
\hline Headache & $65(38.2 \%)$ \\
\hline Tinnitus & $21(12.3 \%)$ \\
\hline
\end{tabular}

Table.5 Fungal isolates obtained in CSOM

\begin{tabular}{|l|c|}
\hline Fungal isolate & No. of cases and percentage \\
\hline Aspergillus flavus & $31(35.6 \%)$ \\
\hline Aspergillus niger & $23(26.4 \%)$ \\
\hline Aspergillus fumigatus & $11(12.6 \%)$ \\
\hline Aspergillus terreus & $2(2.3 \%)$ \\
\hline Candida albicans & $6(6.9 \%)$ \\
\hline Candida species & $4(4.7 \%)$ \\
\hline Penicillium spp & $4(4.7 \%)$ \\
\hline Mucor & $3(3.4 \%)$ \\
\hline Aureobasidium & $2(2.3 \%)$ \\
\hline Phoma Total & $1(1.1 \%)$ \\
\hline \multicolumn{2}{|c|}{ Tom } \\
\hline
\end{tabular}


Table.6 Isolation of fungal pathogen in each age group

\begin{tabular}{|l|c|c|c|c|c|c|c|}
\hline \multicolumn{1}{|c|}{ Age group } & $\mathbf{0 - 1 0}$ & $\mathbf{1 1 - 2 0}$ & $\mathbf{2 1 - 3 0}$ & $\mathbf{3 1 - 4 0}$ & $\mathbf{4 1 - 5 0}$ & $\mathbf{5 1 - 6 0}$ & Total \\
\hline Fungus & & & & & & & \\
\hline Aspergillus flavus & 0 & 0 & 9 & 18 & 3 & 1 & $\mathbf{3 1}$ \\
\hline Aspergillus niger & 0 & 1 & 6 & 13 & 2 & 1 & $\mathbf{2 3}$ \\
\hline Aspergillus fumigatus & 1 & 1 & 5 & 3 & 1 & 0 & $\mathbf{1 1}$ \\
\hline Aspergillus terreus & 0 & 0 & 0 & 1 & 1 & 0 & $\mathbf{2}$ \\
\hline Candida albicans & 0 & 0 & 2 & 2 & 2 & 0 & $\mathbf{6}$ \\
\hline Non Candida albicans & 0 & 0 & 0 & 2 & 2 & 0 & $\mathbf{4}$ \\
\hline Penicillium spp & 0 & 0 & 1 & 1 & 2 & 0 & $\mathbf{4}$ \\
\hline Mucor spp & 0 & 0 & 0 & 1 & 2 & 0 & $\mathbf{3}$ \\
\hline Aureobasidium & 0 & 0 & 1 & 1 & 0 & 0 & $\mathbf{2}$ \\
\hline Phoma & 0 & 0 & 0 & 0 & 1 & 0 & $\mathbf{1}$ \\
\hline \multicolumn{1}{|c|}{ Total } & $\mathbf{1}$ & $\mathbf{2}$ & $\mathbf{2 4}$ & $\mathbf{4 2}$ & $\mathbf{1 6}$ & $\mathbf{2}$ & $\mathbf{8 7}$ \\
\hline
\end{tabular}

Baruah and Agarwal et al., isolated Aspergillus niger, Aspergillus fumigatus, Candida albicans, Candida tropicalis, and Mucor spp. as the causative agents of CSOM. Rodrigues et al., also in his study observed Aspergillus niger as the commonest fungal pathogen. But the studies of Jaiswal observed Candida albicans as the commonest organism causing fungal infection of ear. This finding goes against the present study. Study by Talwar et al., (1988) on fungal infections of ear with special reference to CSOM observed that Aspergillus niger, Aspergillus fumigatus, Aspergillus flavus, Penicillium, Candida albicans, Candida parapsillosis are the most common fungal pathogens.

In conclusion, the mycological study of CSOM reveals Aspergillus species is the most common causative agent followed by Candida. Carefully selected local and/or systemic antibiotics guided by culture and sensitivity, along with the use of frequent ear toilet is an effective treatment modality in general. Long term topical antibiotic therapy, presence of moisture in ear canal etc. can lead to otomycosis. So fungal culture should also done in chronically discharging ear. This will prevent the administration of unwanted antibiotics. Otomycosis is treated by debridment followed with topical azole anti fungals and symptomatically managed with oral antihistamines. Topical $1 \%$ clotrimazole drops yielded highest resolution rate with lowest recurrent rate. Aural toilet with the insertion of gauze saturated in Neomycin, Beclomethazone and Clotrimazole for consecutive three days was very effective for CSOM cases due to fungal infection.

\section{References}

Arya, S.C., Mohapatra, L.N. 1966. Bacteriological and mycotic flora in cases of chronic suppurative otitis media. J. Indian Med. Assoc., 47(8): 369-72.

Bluestone, C.D. 2004. Studies in otitis media; Children's hospital of Pittsburgh. University of Pittsburgh Progress Report. The Laryngoscope, 114: 1-26.

Gulati, Sudesh Kumar. 1997. Investigative profile in patients of chronic suppurative otitis media. Indian J. Otol., 
3(2): 59-62.

Khurana, A.S., Kanta Shashi and Kumar Suresh. 1998. Incidence of fungal infection in CSOM, Indian J. Otol., (3): 121-123.

Klein, J.O. 1998. Microbiology. In: Otitis Media in Infants and Children, 4th ed, Symptoms of acute otitis media. Pediatr. Infect. Dis. J., 17: 676.

Loy, A.H.C., Tan, A.L., Lu, P.K.S. 2002. Microbiology of chronic suppurative otitis media in Singapore. Singapore Med. J., 43(6): 296-99.

Michael Gleeson (Ed). 2008. Scott-Brown's Otorhinolaryngology, Head and Neck Surgery, $7^{\text {th }}$ Edition, Hodder Arnold, Great Britain.

Mohan Urmil, Jindal Neerja. 1998. Fungal and bacterial flora of chronic suppurative otitis media in Amritsar. IJO \& HNS, 50(2): 175-77.

Mohanty, J.C., Mohanty, S.K., Sahoo, R.C., Gosh, S.K. et al. 1999. Clinico microbial profile of otomycosis in Berhampur. Indian J. Otol., 5(2): 81-83.

Murat Ozean, K., Muze ozcan, Aydin Karaarslan Otomycosis in Turkeypredisposing factors, aeiology and therapy. The J. Laryngol. Otol., 117: 3942.

Pankti Panchal, Jayasree Pethani, Dipti Tatel, Sanjay Rathod. 2013. Analysis of various fungal agents in clinically suspected case of otomycosis. Indian J. Basic and Appl. Med. Res., Issue-8, vol2.P:865-869.

Pradhan, B., Ratna, N., Amaya, R.M. 2003. Prevalence of otomycosis in OPD of ENT in Tribhuvan University of Teaching Hospital, Kathmnadu, Nepal. Ann. Otol. Rhinolaryngol., 112: 384387.

Rama Rao, M.V., Jayakar, P.A. 1980. Bacteriological study of chronic suppurative otitis media. Indian J. Med. Assoc., 75: 30-33.

Rippon, J.W. 1986. Med. Mycol., The pathogenic fungi and pathogenic Actinomycetes WB Saunders, $3^{\text {rd }}$ edition.

Stangerup, S.E., Tos, M. 1986. Epidemiology of acute suppurative otitis media, Am. J. Otolaryngo., 7(1): 47-54.

Talwar, P. 1988. Fungal infections of ear with special reference to chronic suppurative Otitis Media. Mycopathologia, 104(1): 47-50.

Vishwanatha, B.D., Sumatha and M.S. Vijayasree Otomycosis inimmuno competant and immune compromised patients; comparative study and literature review Ear, Nose and Throat J., vol 91: P 114-121.

\section{How to cite this article:}

Sahira Haneefa, Sathya Bhama and Jyothi Rajahamsan. 2017. Mycological Profile of Chronic Suppurative Otitis Media in a Tertiary Care Hospital in South India. Int.J.Curr.Microbiol.App.Sci. 6(2): 127-132. doi: http://dx.doi.org/10.20546/ijcmas.2017.602.018 